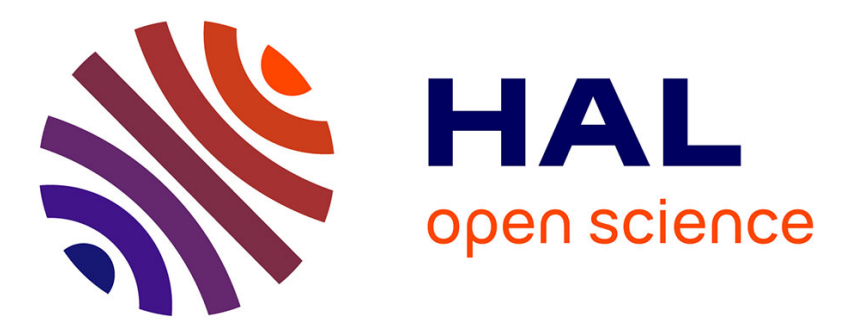

\title{
Formalizing Desargues' theorem in Coq using ranks in Coq
}

\author{
Nicolas Magaud, Julien Narboux, Pascal Schreck
}

\section{To cite this version:}

Nicolas Magaud, Julien Narboux, Pascal Schreck. Formalizing Desargues' theorem in Coq using ranks in Coq. 24th Annual ACM Symposium on Applied Computing, Xiao-Shan Gao, Robert Joan-Arinyo, Dominique Michelucci, Mar 2009, Honolulu, United States. pp.1110-1115, 10.1145/1529282.1529527 . inria-00335719

\section{HAL Id: inria-00335719 https://hal.inria.fr/inria-00335719}

Submitted on 30 Oct 2008

HAL is a multi-disciplinary open access archive for the deposit and dissemination of scientific research documents, whether they are published or not. The documents may come from teaching and research institutions in France or abroad, or from public or private research centers.
L'archive ouverte pluridisciplinaire HAL, est destinée au dépôt et à la diffusion de documents scientifiques de niveau recherche, publiés ou non, émanant des établissements d'enseignement et de recherche français ou étrangers, des laboratoires publics ou privés. 


\section{Formalizing Desargues' theorem in Coq using ranks}

\author{
Nicolas Magaud \\ LSIIT UMR 7005 \\ CNRS-ULP \\ Université de Strasbourg \\ Nicolas.Magaud@dpt- \\ info.u-strasbg.fr
}

\author{
Julien Narboux \\ LSIIT UMR 7005 \\ CNRS-ULP \\ Université de Strasbourg \\ Julien.Narboux@dpt- \\ info.u-strasbg.fr
}

\author{
Pascal Schreck \\ LSIIT UMR 7005 \\ CNRS-ULP \\ Université de Strasbourg \\ Pascal.Schreck@dpt- \\ info.u-strasbg.fr
}

\begin{abstract}
Formalizing geometry theorems in a proof assistant like Coq is challenging. As emphasized in the literature, the nondegeneracy conditions leads to technical proofs. In addition, when considering higher-dimensions, the amount of incidence relations (e.g. point-line, point-plane, line-plane) induce numerous technical lemmas. In this paper, we present an original approach based on the notion of rank which allows to describe incidence and non-incidence relations such as equality, collinearity and coplanarity homogeneously. It allows to carry out proofs in a more systematic way. To validate this approach, we formalize in Coq (using only ranks) one of the fundamental theorems of the projective space, namely Desargues' theorem.
\end{abstract}

\section{Categories and Subject Descriptors}

I.2.4 [COMPUTING METHODOLOGIES]: Knowledge Representation Formalisms and Methods; G.4 [MATHEMATICAL SOFTWARE]: Certification and testing

\section{Keywords}

formalization, Desargues, rank, projective geometry, Coq

\section{INTRODUCTION}

This paper deals with formally proving in Coq Desargues' theorem in at least 3-dimensional projective geometry using the concept of rank. In the longer term, the underlying objective of the presented work consists in designing a geometric formal prover able to handle the non-degeneracy conditions in geometry, and especially in geometric constraint solving $[12,11]$.

Indeed, when designing an object by the mean of geometric constraints, one has to correctly and completely specify the shape of the object. The corresponding constraint system can lead to contradictions and degenerate cases which

* partially supported by the research project Galapagos.

This is the author's version of the work. It is posted here by permission of ACM for you personal use. Not for redistribution. The definitive version was published in the proceedings of SAC 2009.

SAC'09 March 8-12, 2009, Honolulu, Hawaii, U.S.A.

Copyright 2009 ACM 978-1-60558-166-8/09/03 ...\$5.00. must be handled properly by the solver as shown in [20]. The structural contradictions are easy to detect, whereas those resulting from geometric theorems are more difficult to deal with.

We focus on projective geometry which is a simple but powerful enough setting to express arbitrarily complex problems as shown in [16]. Moreover, in 3D (or higher) proofs become much more difficult than in 2D: first, Desargues' property becomes a theorem and consequently all the projective spaces arise from a division field; second incidence geometry has to deal not only with points and lines, but also with planes or, more generally, flats; third there is a combinatoric explosion of cases.

We propose to use the concept of rank, which provides a generic way to describe incidence relations. Informally, ranks allow to distinguish between equal/nonequal points, collinear/non-collinear points, coplanar/noncoplanar points, etc. To validate this approach, we carry out a mechanized proof (using only ranks) of one of the fundamental theorems of the projective space, namely Desargues' theorem.

Related work Proof assistants have already been used in the context of geometry. Numerous papers have emphasized the importance of the problem of degenerate cases in formal geometry [8, 15, 10, 19]. Brandt and Schneider studied how to handle degenerate cases for the orientation predicates in computational geometry using three valued logic [3]. Bezem and Hendricks formalized Hessenberg's theorem in Coq [2]. Guilhot has formalized in Coq a proof of Desargues' theorem in affine geometry [10]. Narboux has formalized in Coq the area method of Chou, Gao and Zhang [5, 18] and applied it to obtain a proof of Desargues' theorem in affine geometry. Kusak has formalized in Mizar Desargues' theorem in the Fanoian projective at least 3-dimensional space [13]. The assumption that the space is Fanoian makes the theorem more specialized than ours. We have performed a formalization of projective plane geometry in Coq [14]. The idea of proving projective space theorems with ranks is suggested by Michelucci and Schreck in [17].

Outline The paper is organized as follows. In section 2, we present the axioms for the projective at least 3-dimensional space. In section 3, we introduce the concept of rank and another axiom system to capture projective geometry using ranks. We show how non-degeneracy conditions can be expressed nicely using the notion of rank. In section 4 , we propose a proof of Desargues' theorem purely based on the concept of rank. In section 5 , we present the mechanization of the proof using the Coq proof assistant $[1,6]$. 


\section{AXIOM SYSTEM}

Projective geometry is a general setting in the hierarchy of geometries which assumes that two lines in a plane always meet $[7,4]$. We first assume that we have two kinds of objects (points and lines) ${ }^{1}$. We then consider a relation $(\in)$ between elements of these two sets. The following axioms describe projective at least 3-dimensional space:

Line-Existence $\forall A B$ : Point, $\exists l$ : Line, $A \in l \wedge B \in l$

$\forall A B C D:$ Point, $\forall l_{A B} l_{C D} l_{A C} l_{B D}:$ Line, $A \neq B \wedge A \neq C \wedge A \neq D \wedge B \neq C \wedge B \neq D \wedge C \neq D \wedge$

Pasch $A \in l_{A B} \wedge B \in l_{A B} \wedge C \in l_{C D} \wedge D \in l_{C D} \wedge$

$A \in l_{A C} \wedge C \in l_{A C} \wedge B \in l_{B D} \wedge D \in l_{B D} \wedge$

$\left(\exists I:\right.$ Point,$\left.I \in l_{A B} \wedge I \in l_{C D}\right) \Rightarrow$

$\left(\exists J:\right.$ Point,$\left.J \in l_{A C} \wedge J \in l_{B D}\right)$

Three-Points $\forall l:$ Line, $\exists A B C$ : Point, $A \neq B \wedge B \neq C \wedge A \neq C \wedge A \in l \wedge B \in l \wedge C \in l$

Uniqueness $\forall A B$ : Point, $\forall l m$ : Line, $A \in l \wedge B \in l \wedge A \in m \wedge B \in m \Rightarrow A=B \vee l=m$

Lower-Dimension $\exists l m:$ Line, $\forall p:$ Point, $p \notin l \vee p \notin m$

Pasch's axiom ensures that two co-planar lines always meet. We presented this standard axiom system as a reference, but to ease the formalization in Coq, we propose an alternative axiom system based on the notion of rank.

\section{RANKS}

The concept of rank is a general notion of matroid theory. An integer function $r k$ on $E$ is the rank function of a matroid if and only if the following conditions are satisfied:

R1 $\forall X \subseteq E, 0 \leq r k(X) \leq|X|$ (nonnegative and subcardinal)

R2 $\forall X Y \subseteq E, X \subseteq Y \Rightarrow r k(X) \leq r k(Y) \quad$ (nondecreasing)

R3 $\forall X Y \subseteq E, r k(X \cup Y)+r k(X \cap Y) \leq r k(X)+r k(Y)$ (submodular)

In projective geometry, we can define a rank function on sets of points which verify the axioms above: a flat being a set of points closed by the collinearity relation, the rank of a set of points $A$ is the cardinal of a smallest set generating $A$ (see figure 1 for examples).

$$
\begin{array}{ll}
r k\{A, B\}=1 & A=B \\
r k\{A, B\}=2 & A \neq B \\
r k\{A, B, C\}=2 & A, B, C \text { are collinear } \\
& \text { with at least two of them dist } \\
r k\{A, B, C\} \leq 2 & A, B, C \text { are collinear } \\
r k\{A, B, C\}=3 & A, B, C \text { are not collinear } \\
r k\{A, B, C, D\}=3 & A, B, C, D \text { are co-planar, } \\
& \text { not all collinear } \\
r k\{A, B, C, D\}=4 & A, B, C, D \text { are not co-planar } \\
r k\{A, B, C, D, E\} \leq 2 & A, B, C, D, E \text { are all collinear }
\end{array}
$$

Figure 1: Rank statements and their geometric interpretations

\footnotetext{
${ }^{1}$ Planes are not basic objects in this axiom system, but are defined within the theory.
}

Using this definition, one can show that every projective space has a matroid structure, but the converse is not true. In the next section, we introduce additional axioms to capture 3D or higher projective geometry.

\subsection{A rank-based axiom system}

Contrary to the axiom system shown in section 2, we assume that we have only one kind of objects, namely points. To capture the whole projective space, we need to add some new axioms to the matroid's ones:

$$
\begin{aligned}
& \text { Rk-Singleton } \forall P: \text { Point }, r k\{P\} \geq 1 \\
& \text { Rk-Couple } \forall P Q: \text { Point }, P \neq Q \Rightarrow r k\{P, Q\} \geq 2 \\
& \text { Rk-Pasch } \forall A B C D, r k\{A, B, C, D\} \leq 3 \Rightarrow \\
& \quad \exists J, r k\{A, B, J\}=r k\{C, D, J\}=2 \\
& \text { Rk-Three-Points } \quad \forall A B, \exists C, r k\{A, B, C\}=r k\{B, C\}=r k\{A, C\}=2 \\
& \text { Rk-Lower-Dimension } \exists A B C D, r k\{A, B, C, D\} \geq 4
\end{aligned}
$$

The first two ones ensure that the rank function is not degenerate. Rk-Pasch is the translation of Pasch's axiom: $r k\{A, B, C, D\} \leq 3$ means these points are coplanar, thus that the two lines $A B$ and $C D$ intersect.

Using this axiom system we formally proved all the axioms of section 2. In particular the following Rk-Uniqueness lemma is derivable and can be used to prove the Uniqueness axiom:

Lemma 1 (RK-Uniqueness).

$$
\forall A B C D M P,\left(\begin{array}{c}
r k\{A, B\}=2 \\
r k\{C, D\}=2 \\
r k\{A, B, M\} \leq 2 \\
r k\{C, D, M\} \leq 2 \\
r k\{A, B, P\} \leq 2 \\
r k\{C, D, P\} \leq 2 \\
r k\{A, B, C, D\} \geq 3
\end{array}\right) \Rightarrow r k\{M, P\}=1
$$

Proof. See section 6.

We can also derive a lemma which expresses concisely that for every point there exists one which is different, for every line there exists a point not on this line and for every plane there exists a point not on this plane:

$$
\begin{aligned}
& \text { Lemma } 2 \text { (Construction). } \\
& \quad \forall E, r k(E) \leq 3 \Rightarrow \exists P, r k(E \cup\{P\})=r k(E)+1
\end{aligned}
$$

Overall, this axiom system is convenient as first it only deals with points and hence the theory is dimension-independent, second ranks allow to summarize both positive and negative assumptions about sets of points homogeneously.

\subsection{Proof techniques using ranks}

In this section we describe two proofs techniques which are simple but important to simplify formal proofs.

First, all equalities about ranks (say $r k(a)=r k(b)$ ) are usually proved in two steps: first showing that $r k(a) \leq r k(b)$ and then that $r k(a) \geq r k(b)$. Consequently, when stating a lemma, it is worth being cautious about whether the actual equality is required or if one of the two inequalities is enough to go on with the proofs. This approach allows to avoid 
numerous technical lemmas when carrying out the formal proofs in the Coq proof assistant.

Second, in the proving process, we make often use of axiom R3. For instance, if we need to prove a statement like:

$$
r k\{A, B, C, D, I\}+r k\{I\} \leq r k\{A, B, I\}+r k\{C, D, I\}
$$

we could be tempted to instantiate axiom R3 with $X:=$ $\{A, B, I\}$ and $Y:=\{C, D, I\}$. But unfortunately, this statement is not a direct consequence of axiom R3. For instance $A$ may be equal to $C$ and consequently $\{A, B, I\} \cap$ $\{C, D, I\}=\{A, I\}$. Determining the intersection of two finite sets of points requires to distinguish cases about the equality of these points. This leads to intricate proofs in Coq. Therefore, in the rest of this paper, we shall never consider the real set theoretical intersection but a lower approximation of the intersection (noted $\sqcap$ ).

Definition 1 (Literal intersection). Let $L_{1}$ and $L_{2}$ be two sets of points. By definition $L_{1} \sqcap L_{2}$ is the intersection of the two sets of points considered syntactically.

Using literal intersection we can derive a more convenient version of axiom R3 which leads to fewer case distinctions:

Lemma 3 (R3-Lit).

$$
\forall X Y, r k(X \cup Y)+r k(X \sqcap Y) \leq r k(X)+r k(Y)
$$

In Coq, it is not possible to define the litteral intersection. To capture the intent of this lemma we rather use:

Lemma 4 (R3-ALt).

$\forall X Y I, I \subseteq X \cap Y \Rightarrow r k(X \cup Y)+r k(I) \leq r k(X)+r k(Y)$

This lemma will be used heavily in the next sections.

\section{DESARGUES' THEOREM}

Desargues' theorem states that:

let $E$ be a $3 D$ or higher projective space and $A, B, C, A^{\prime}, B^{\prime}, C^{\prime}$ be points in $E$, if the three lines joining the corresponding vertices of triangles $A B C$ and $A^{\prime} B^{\prime} C^{\prime}$ all meet in a point $O$, then the three intersections of pairs of corresponding sides $\alpha, \beta$ and $\gamma$ lie on a line.

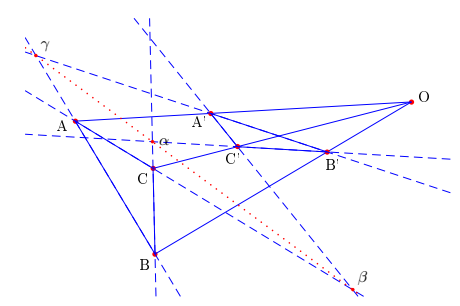

Even though it can be expressed, it is not provable when $\mathrm{E}$ is a plane (this is coherent with the fact that there are non-Desarguesian projective planes).

\subsection{Proving Desargues' Theorem}

The idea of the proof is classic: we first prove a version of the theorem where the two triangles are not coplanar, we call it Desargues 3D (see section 4.2) and then we deduce from it a version where $A, B, C, A^{\prime}, B^{\prime}$ and $C^{\prime}$ lie on a same plane (Desargues 2D) as shown on figure 2 .
As we will see in the next section, using the concept of rank the proof of the $3 \mathrm{D}$ version is straightforward and special cases can be handled smoothly. In section 4.3 , we will show how we actually build the $2 \mathrm{D}$ version and conclude the proof of the original theorem.

\subsection{A 3D version of Desargues' Theorem}

In this section, we prove Desargues' 3D theorem.

Theorem 1 (Desargues 3D). Let's consider two (non degenerate) triangles $A B C$ and abc such that they are perspective from a given point $O$ :

$$
\begin{gathered}
r k\{A, B, C\}=r k\{a, b, c\}=3 \\
r k\{a, A, O\}=r k\{b, B, O\}=r k\{c, C, O\}=2
\end{gathered}
$$

We assume this forms a non planar figure:

$$
r k\{A, B, C, a, b, c\} \geq 4
$$

and define three points $\alpha, \beta, \gamma$ such that:

$$
\begin{aligned}
& r k\{A, B, \gamma\}=r k\{a, b, \gamma\}=2 \\
& r k\{A, C, \beta\}=r k\{a, c, \beta\}=2 \\
& r k\{B, C, \alpha\}=r k\{b, c, \alpha\}=2
\end{aligned}
$$

Under these assumptions, $r k\{\alpha, \beta, \gamma\} \leq 2$ holds.

Proof of Desargues 3D.

Lemma 5. $r k\{A, B, C, \alpha\}=3$

Proof. By assumption $r k\{A, B, C\}=3$, hence using axiom R2, $r k\{A, B, C, \alpha\} \geq 3$. Moreover using R3-ALT we have:

$$
\begin{aligned}
r k\{A, B, C, \alpha\}+r k\{B, C\} & \leq r k\{A, B, C\}+r k\{\alpha, B, C\} \\
r k\{A, B, C, \alpha\}+2 & \leq 3+2
\end{aligned}
$$

Hence, we can conclude that $r k\{A, B, C, \alpha\}=3$. Similar proofs can be done with $\beta$ and $\gamma$.

Lemma 6. $r k\{A, B, C, \alpha, \beta\}=3$

Proof. First, using axiom R2 and lemma 5 we have $r k\{A, B, C, \alpha, \beta\} \geq 3$. Second, using R3-ALt we have: $r k\{A, B, C, \alpha, \beta\}+r k\{A, B, C\}$

$r k\{A, B, C, \alpha, \beta\}+3 \leq 3+3$

$$
\leq r k\{A, B, C, \alpha\}+r k\{A, B, C, \beta\}
$$

Hence, we can conclude that $r k\{A, B, C, \alpha, \beta\}=3$.

Lemma 7. $r k\{A, B, C, \alpha, \beta, \gamma\}=r k\{a, b, c, \alpha, \beta, \gamma\}=3$

Proof. The proof is similar to lemma 6.

Lemma 8. $r k\{A, B, C, a, b, c, \alpha, \beta, \gamma\} \geq 4$

Proof. By assumption $r k\{A, B, C, a, b, c\} \geq 4$, hence using axiom $\mathrm{R} 2, r k\{A, B, C, a, b, c, \alpha, \beta, \gamma\} \geq 4$.

Using these lemmas we can conclude the proof:

From R3-ALT we know that:

$$
\begin{aligned}
r k\{A, B, C, a, b, c, \alpha, \beta, \gamma\}+r k\{\alpha, \beta, \gamma\} \\
\leq r k\{A, B, C, \alpha, \beta, \gamma\}+r k\{a, b, c, \alpha, \beta, \gamma\}
\end{aligned}
$$

Hence, using lemmas 7 and $8 r k\{\alpha, \beta, \gamma\} \leq 2$ holds.

\subsection{Lifting from 2D to $3 D$}

In this section, we prove the $2 \mathrm{D}$ version of Desargues using the $3 \mathrm{D}$ version. 


\subsubsection{Statement}

Most assumptions are the same as in the 3D version. Let's consider two triangles $A B C$ and $A^{\prime} B^{\prime} C^{\prime}$ such that they are perspective from a given point $O$ :

$$
\begin{gathered}
r k\{A, B, C\}=r k\left\{A^{\prime}, B^{\prime}, C^{\prime}\right\}=3 \\
r k\left\{A^{\prime}, A, O\right\}=r k\left\{B^{\prime}, B, O\right\}=r k\left\{C^{\prime}, C, O\right\}=2
\end{gathered}
$$

We define three points $\alpha, \beta, \gamma$ such that:

$$
\begin{aligned}
& r k\{A, B, \gamma\}=r k\left\{A^{\prime}, B^{\prime}, \gamma\right\}=2 \\
& r k\{A, C, \beta\}=r k\left\{A^{\prime}, C^{\prime}, \beta\right\}=2 \\
& r k\{B, C, \alpha\}=r k\left\{B^{\prime}, C^{\prime}, \alpha\right\}=2
\end{aligned}
$$

Contrary to the $3 \mathrm{D}$ case, we assume this forms a planar figure:

$$
r k\left\{A, B, C, A^{\prime}, B^{\prime}, C^{\prime}, O\right\}=3
$$

In addition to these assumptions which are closely related to those of Desargues' 3D theorem, the following nondegeneracy conditions are required:

$$
\begin{gathered}
r k\{A, B, O\}=r k\{A, C, O\}=r k\{B, C, O\}=3 \\
r k\left\{A, A^{\prime}\right\}=r k\left\{B, B^{\prime}\right\}=r k\left\{C, C^{\prime}\right\}=2
\end{gathered}
$$

Desargues' theorem states that, under these assumptions, $r k\{\alpha, \beta, \gamma\} \leq 2$ holds.

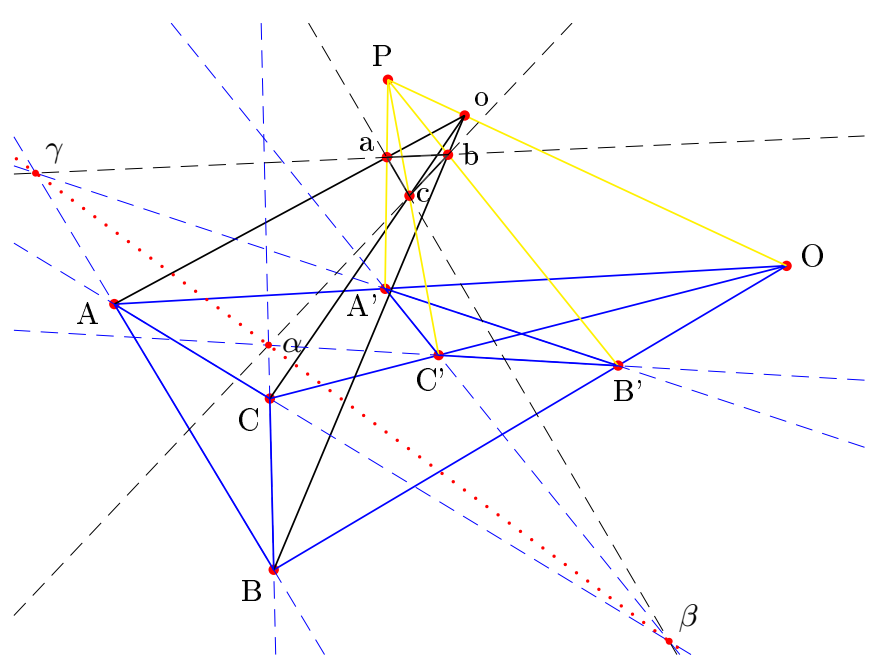

Figure 2: Desargues' theorem (3D extrusion) $A, B, C, A^{\prime}, B^{\prime}, C^{\prime}, O, \alpha, \beta$ and $\gamma$ are co-planar. If $P$ is the sun, triangle $a b c$ then casts its shadow in $A^{\prime} B^{\prime} C^{\prime}$.

\subsubsection{Informal Proof}

We have to lift triangle $A^{\prime} B^{\prime} C^{\prime}$ into a new triangle $a b c$ which is not coplanar with triangle $A B C$ in order to have a configuration of points in which Desargues' 3D theorem can be applied. The construction is shown in figure 2 . Here are the main steps: we first construct a point $P$ which lies outside the plane formed by $A, B, C, A^{\prime}, B^{\prime}, C^{\prime}$ and $O$. We know such a point $P$ exists thanks to lemma 2 . We then build a line incident to $P$ and $O$ (the point from which triangles $A B C$ and $A^{\prime} B^{\prime} C^{\prime}$ are perspective) and consider a third point $O$ on this line (axiom Rk-Three-Points ensures such a point exists and is different from both $O$ and $P)$. We construct a new point $a$ as the intersection of lines $P A^{\prime}$ and $o A$. We know these two lines intersect because of
Pasch's axiom and the fact that lines $A A^{\prime}$ and $P o$ intersect in $O$. We do the same to construct points $b$ and $c$. Applying Desargues' $3 \mathrm{D}$ theorem to $A B C$ and $a b c$ requires to make sure we have a non-degenerate $3 \mathrm{D}$ figure and that $a b c$ is a non-degenerate triangle. We also have to make sure $\alpha$ defined as the intersection of lines $B C$ and $B^{\prime} C^{\prime}$ is the same as the $\alpha$ of Desargues' 3D theorem which is the intersection of $B C$ and $b c$. This requirement can be satisfied by simply proving that $\alpha$ is incident to $b c$. The same requirement applies for $\beta$ and $\gamma$. Overall, we have to prove the following statements which are requirements to apply Desargues' $3 \mathrm{D}$ version (proofs are given in appendix B). Note that when applying the theorem, the point $O$ plays the role of point $O$.

$$
\begin{gathered}
r k\{A, B, C\}=r k\{a, b, c\}=3 \\
r k\{A, B, C, a, b, c\} \geq 4 \\
r k\{a, b, \gamma\}=r k\{a, c, \beta\}=r k\{b, c, \alpha\}=2 \\
r k\{A, B, \gamma\}=r k\{A, C, \beta\}=r k\{B, C, \alpha\}=2
\end{gathered}
$$

\subsubsection{General Lemmas}

Most proofs are fairly technical, simply using the matroid axioms of rank. However, some lemmas can be highlighted, especially for their genericity and their pervasive use throughout the proofs. Among them, some stability lemmas state that one of the points of a set characterizing a flat (e.g a plane or the whole space) can be replaced by another one belonging to this flat.

$$
\begin{aligned}
& \text { Lemma } 9 \text { (Plane Representation Change). } \\
& \left(\begin{array}{c}
r k\{A, B, C\}=3 \\
r k\{A, B, C, M\}=3 \\
r k\{B, C, M\}=3 \\
r k\{A, B, C, P\}=4
\end{array}\right) \Rightarrow r k\{M, B, C, P\}=4
\end{aligned}
$$

This lemma is heavily used to prove all possible statements expressing that $P$ lies outside the plane formed by $A, B, C, A^{\prime}, B, C^{\prime}, O$.

Other lemmas about coplanarity and also upper bound on ranks when merging a plane and a line are convenient as well. They could form the basis of an automation procedure when doing computer-checked formal proofs.

\section{FORMALIZATION IN COQ}

We mechanize the proof of Desargues' theorem using the Coq proof assistant which implements a higher order intuitionistic logic based on type theory. In such a proof assistant, every step of reasoning is proposed by the user but checked by the system. It dramatically increases the reliability of the proofs compared to paper-and-pencil proofs. In addition, during the development process, being able to change the axiom system easily is very convenient. Proofs can be automatically rechecked by the system and changes only require minor rewriting of the proofs.

The formalization in Coq of our axiom system is quite straightforward ${ }^{2}$. To increase reusability of the proofs we define it as a module type of Coq's module system (see figure 3). This module depends on DecPoints which defines the type of points with a decidable equality.

In Coq, Desargues' theorem can be stated as follows: Theorem Desargues_ $x D^{\prime}$ :

$\forall A^{\prime} B^{\prime} C^{\prime} A B C$ O alpha beta gamma: Point, $r k($ triple $A B C)=3 \rightarrow$ rk $\left(\right.$ triple $\left.A^{\prime} B^{\prime} C^{\prime}\right)=3 \rightarrow$

${ }^{2}$ For lack of space, some technical details are omitted here. 
Module Type RankProjectiveSpace (DecPoints: UsualDecidableType).

Parameter $r k$ : set_of_points $\rightarrow$ nat.

Axiom matroid1_a $: \forall X, r k X \geq 0$.

Axiom matroid1_ $b: \forall X, r k X \leq$ cardinal $X$.

Axiom matroid2: $\forall X Y$, Subset $X Y \rightarrow r k X \leq r k Y$

Axiom matroid3: $\forall X Y$,

$r k($ union $X Y)+r k($ inter $X Y) \leq r k X+r k Y$.

Axiom rk_singleton $: \forall P, r k$ (singleton $P) \geq 1$.

Axiom rk_couple $: \forall P Q, P \neq Q \rightarrow$ rk (couple $P Q) \geq 2$.

Axiom pasch : $\forall A B C \mathrm{D}, r k$ (quadruple $A B C D) \leq 3 \rightarrow$

$\exists J$, rk (triple A $B J)=2 \wedge$ rk (triple $C D J)=2$.

Axiom three_points : $\forall A B, \exists C$,

rk (triple $A B C)=2 \wedge$ rk (couple $B C)=2 \wedge r k$ (couple $A C)=2$.

Parameter P0 P1 P2 P3: Point.

Axiom lower_dim : rk (quadruple P0 P1 P2 P3) $\geq 4$.

End RankProjectiveSpace.

Figure 3: Definition of projective space geometry with ranks in Coq

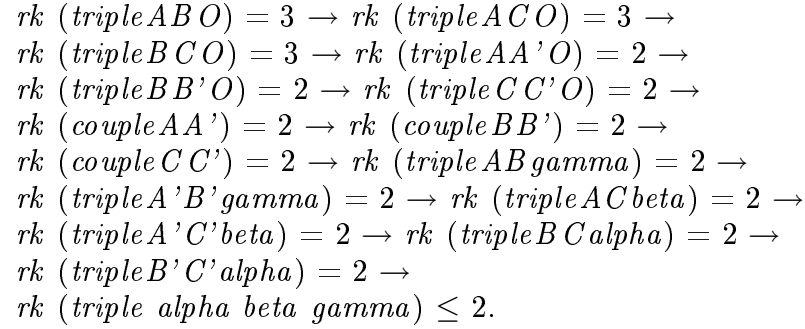

On the technical side, defining our axiom system based on ranks requires a formal description of the concept of sets of points. As our development manipulates only finite sets, we use the development FSets of Filliâtre and Letouzey [9]. Since the provided set equality differs from standard (Leibniz) Coq equality, we have to prove that $r k$ is a morphism with respect to set equality.

Overall, our development consists of more than 6000 lines and 200 lemmas and their formal proofs organized as shown in the figures below. The proof of Desargues' theorem in itself is relatively concise because we proved some generic lemmas and reused them efficiently taking advantage of the symmetries in the statement of Desargues' theorem.

\begin{tabular}{|c|c|c|c|c|}
\hline & 3D & 2D & Framework & Total \\
\hline lines of Coq specs & 215 & 450 & 777 & 1442 \\
\hline lines of Coq proofs & 963 & 976 & 2331 & 4670 \\
\hline
\end{tabular}

\section{CONCLUSIONS}

We proposed a new way to express nicely incidence relations in a 3D setting thanks to ranks and designed an axiom system to capture projective geometry using ranks. We successfully applied it to prove Desargues' theorem. We presented proof engineering techniques which allow to have proofs of reasonable size. In the future, we plan to study how the notion of rank can be used to automatically derive incidence properties. We believe that the genericity of the notation will help the automation process.

Availability The full Coq development is available at the following url: http://galapagos.gforge.inria.fr/
Acknowledgments We wish to thank Negar Gholamizadeh Behbahani who studied some lemmas during an internship.

\section{REFERENCES}

[1] Y. Bertot and P. Castéran. Interactive Theorem Proving and Program Development, Coq'Art: The Calculus of Inductive Constructions. Springer, 2004.

[2] M. Bezem and D. Hendriks. On the Mechanization of the Proof of Hessenberg's Theorem in Coherent Logic. J. of Automated Reasoning, 40(1):61-85, 2008.

[3] J. Brandt and K. Schneider. Using three-valued logic to specify and verify algorithms of computational geometry. In ICFEM, volume 3785 of $L N C S$, pages 405-420. Springer-Verlag, 2005.

[4] F. Buekenhout, editor. Handbook of Incidence Geometry. North Holland, 1995.

[5] S.-C. Chou, X.-S. Gao, and J.-Z. Zhang. Machine Proofs in Geometry. World Scientific, 1994.

[6] Coq development team. The Coq Proof Assistant Reference Manual, Version 8.2. LogiCal Project, 2008.

[7] H. S. M. Coxeter. Projective Geometry. Springer, 1987.

[8] C. Dehlinger, J.-F. Dufourd, and P. Schreck. Higher-Order Intuitionistic Formalization and Proofs in Hilbert's Elementary Geometry. In $A D G^{\prime} 00$, volume 2061 of $L N A I$, pages 306-324. Springer-Verlag, 2000.

[9] J.-C. Filliâtre and P. Letouzey. Functors for Proofs and Programs. In ESOP'2004, volume 2986 of LNCS, pages 370-384. Springer-Verlag, 2004.

[10] F. Guilhot. Formalisation en Coq et visualisation d'un cours de géométrie pour le lycée. TSI, 24:1113-1138, 2005. In french.

[11] C. M. Hoffmann and R. Joan-Arinyo. Handbook of Computer Aided Geometric Design, chapter Parametric Modeling, pages 519-541. Elsevier, 2002.

[12] C. Jermann, G. Trombettoni, B. Neveu, and P. Mathis. Decomposition of geometric constraint systems: a survey. International J. of Computational Geometry and Application, 16(5-6):379-414, 2006.

[13] E. Kusak. Desargues theorem in projective 3-space. $J$. of Formalized Mathematics, 2, 1990.

[14] N. Magaud, J. Narboux, and P. Schreck. Formalizing Projective Plane Geometry in Coq. accepted for presentation at ADG'08, September 2008.

[15] L. Meikle and J. Fleuriot. Formalizing Hilbert's Grundlagen in Isabelle/Isar. In Theorem Proving in Higher Order Logics, pages 319-334, 2003.

[16] D. Michelucci, S. Foufou, L. Lamarque, and P. Schreck. Geometric constraints solving: some tracks. In SPM '06, pages 185-196. ACM Press, 2006.

[17] D. Michelucci and P. Schreck. Incidence Constraints: a Combinatorial Approach. International J. of Computational Geometry and Application, 16(5-6):443-460, 2006.

[18] J. Narboux. A decision procedure for geometry in Coq. In TPHOLs' 04 , volume 3223 of $L N C S$, pages 225-240. Springer-Verlag, 2004.

[19] J. Narboux. Mechanical theorem proving in Tarski's geometry. In $A D G^{\prime} 06$, volume 4869 of $L N A I$, pages 139-156. Springer-Verlag, 2007.

[20] P. Schreck. Robustness in CAD Geometric Construction. In $I V^{\prime} 01$, pages 111-116, London, 2001. 


\section{APPENDIX}

\section{A. PROOFS RELEVANT TO THE FIRST SECTION}

Lemma 10 (Rk-Uniqueness).

$\forall A B C D M P,\left(\begin{array}{c}r k\{A, B\}=2 \\ r k\{C, D\}=2 \\ r k\{A, B, M\} \leq 2 \\ r k\{C, D, M\} \leq 2 \\ r k\{A, B, P\} \leq 2 \\ r k\{C, D, P\} \leq 2 \\ r k\{A, B, C, D\} \geq 3\end{array}\right) \Rightarrow r k\{M, P\}=1$

Proof. Using R3-ALT we have:

$r k\{A, B, M, P\}+r k\{A, B\} \leq r k\{A, B, M\}+r k\{A, B, P\}$

Hence $r k\{A, B, M, P\}=2$, similarly we can show that $r k\{C, D, M, P\}=2$. Moreover $r k\{A, B, C, D, M, P\} \geq 3$ as $r k\{A, B, C, D\} \geq 3$ and $\{A, B, C, D\} \subseteq\{A, B, C, D, M, P\}$. Finally, using R3-ALT we have: $r k\{A, B, C, D, M, P\}+r k\{M, P\}$

$$
\leq r k\{A, B, M, P\}+r k\{C, D, M, P\}
$$

Hence: $3+r k\{M, P\} \leq 2+2$.

Our development is based on matroid axioms R1, R2 and R3. But one can prove that they are equivalent to the following set of axioms:

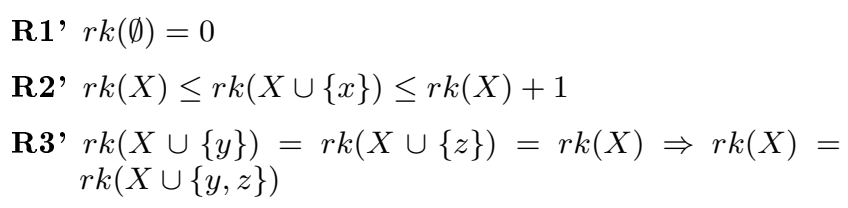

\section{Lemma 11 (Lower Dimension).}

$$
\exists A B C D, \forall M, r k\{A, B, M\} \neq 2 \vee r k\{C, D, M\} \neq 2
$$

Proof. Using axiom Rk-Lower-Dimension, we obtain $A, B, C$ and $D$ such that $r k\{A, B, C, D\}=4$.

Suppose that $r k\{A, B, M\}=2$ and $r k\{C, D, M\}=2$.

Using R3-ALT we have that:

$r k\{A, B, C, D, M\}+r k\{M\} \leq r k\{A, B, M\}+r k\{C, D, M\}$

Hence $r k\{A, B, C, D, M\} \leq 3$, which is in contradiction with $r k\{A, B, C, D\}=4$.

\section{Lemma 12 (Construction).}

$$
\forall E, r k(E) \leq 3 \Rightarrow \exists P, r k(E \cup\{P\})=r k(E)+1
$$

Proof. Consider $E$ such that $r k(E) \leq 3$. Using axiom Rk-Lower-Dimension we obtain $A, B, C$ and $D$ such that $r k\{A, B, C, D\}=4$. Using R2' we know that $r k(E) \leq$ $r k(E \cup\{A\}) \leq r k(E)+1$ and similarly for $B, C$ and $D$. Suppose that $r k(E \cup\{A\})=r k(E \cup\{B\})=r k(E \cup$ $\{C\})=r k(E \cup\{D\})=r k(E)$, then we would obtain $r k(E \cup\{A, B, C, D\})=r k(E)$ by repeated applications of R3'. This is in contradiction with $r k\{A, B, C, D\}=4$ since $r k(E) \leq 3$. Hence there exists a $P$ such that $r k(E \cup\{P\})=$ $r k(E)+1$.

\section{B. PROVING DESARGUES' 2D THEOREM}

We remind the reader of the properties we need to prove in order to apply Desargues' 3D theorem, namely:

$$
\begin{gathered}
r k\{A, B, C\}=r k\{a, b, c\}=3 \\
r k\{A, B, C, a, b, c\} \geq 4 \\
r k\{a, b, \gamma\}=r k\{a, c, \beta\}=r k\{b, c, \alpha\}=2 \\
r k\{A, B, \gamma\}=r k\{A, C, \beta\}=r k\{B, C, \alpha\}=2
\end{gathered}
$$

Statements $r k\{A, B, \gamma\}=r k\{A, C, \beta\}=r k\{B, C, \alpha\}=2$ and $r k\{A, B, C\}=3$ are assumptions of the Desargues' $2 \mathrm{D}$ theorem, therefore their proofs are immediate.

\section{B.1 Preliminary Lemmas}

We remind the reader that $A, B, C, A^{\prime}, B^{\prime}, C^{\prime}$ and $O$ lie in the same plane. $P$ is a point outside this plane. $O$ is a third point on the line $O P$. The point $a$ is defined as the intersection of lines $P A^{\prime}$ and $o A$. Points $b$ and $c$ are defined in a similar way. In this setting, the following lemmas hold:

Lemma 13. $r k\left\{A^{\prime}, B^{\prime}, O\right\}=r k\left\{A^{\prime}, C^{\prime}, O\right\}=r k\left\{B^{\prime}, C^{\prime}, O\right\}=3$

Lemma 14. $r k\left\{A^{\prime}, B^{\prime}, O, P\right\}=r k\left\{A^{\prime}, B^{\prime}, O, o\right\}=4$

Lemma 15. $r k\{A, B, O, a\} \geq 4 \quad r k\left\{A, A^{\prime}, C, a\right\} \geq 4$

Lemma 16. $r k\{A, B, O, b\} \geq 4 \quad r k\{A, B, O, c\} \geq 4$

LEMMA 17. $r k\{o, a\}=r k\{o, b\}=r k\{o, c\}=2$

Lemma 18. $r k\{a, c, A, C, \beta\}=r k\left\{a, c, A^{\prime}, C^{\prime}, \beta\right\}=3$

\section{B.2 Proving Desargues' 3D assumptions}

Lemma 19.

$$
r k\{A, B, C, a, b, c\} \geq 4
$$

Proof. By lemma 16, we have $r k\{A, B, O, b\}>=4$, hence $r k\{A, B, C, O, b\} \geq 4$. Using axiom R3-ALT, we have: $r k\{A, B, C, b\}+r k\{A, B, C, O\}$

$r k\{A, B, C, b\}+3 \geq 4+3$

$$
\geq r k\{A, B, C, O, b\}+r k\{A, B, C\}
$$

Consequently we have $r k\{A, B, C, b\} \geq 4$ and applying axiom R2 twice, it leads to $\operatorname{rk}\{A, B, C, a, b, c\} \geq 4$.

Lemma 20. $r k\{a, b, c\}=3$

Proof. By axiom R1 we have $r k\{a, b, c\} \leq 3$.

Let's prove $r k\{a, b, c\} \geq 3$.

By axiom R3-ALT, we have:

$r k\{a, b, c, o, A, B\}+r k\{o, C, c\}$

$$
\geq r k\{A, B, C, o, a, b, c\}+r k\{o, c\}
$$

$r k\{a, b, c, o, A, B\}+2 \geq 4+2$, hence $r k\{a, b, c, o, A, B\} \geq 4$. Again, using axiom R3-ALT we have:

$r k\{a, b, c, o, A\}+r k\{o, B, b\} \geq r k\{a, b, c, o, A, B\}+r k\{o, b\}$

$r k\{a, b, c, o, A\}+2>=4+2$, hence $r k\{a, b, c, o, A\} \geq 4$.

Applying axiom R3-ALT one last time yields:

$r k\{a, b, c\}+r k\{o, A, a\} \geq r k\{a, b, c, o, A\}+r k\{a\}$

$r k\{a, b, c\}+2 \geq 4+1$, hence $r k\{a, b, c\} \geq 3$.

Note that this proof relies on the facts that $r k\{o, b\}=2$ and $r k\{o, c\}=2$ which are proved as lemma 17 .

Lemмa 21. $r k\{a, b, \gamma\}=r k\{a, c, \beta\}=r k\{b, c, \alpha\}=2$

Proof. Using axiom R3-ALt, we have:

$r k\{a, c, A, C, \beta\}+r k\left\{a, c, A^{\prime}, C^{\prime}, \beta\right\}$

$$
\geq r k\left\{a, c, A, C, A^{\prime}, C^{\prime}, \beta\right\}+r k\{a, c, \beta\}
$$

We have $r k\left\{a, c, A, C, A^{\prime}, C^{\prime}, \beta\right\} \geq 4$ using axiom $\mathrm{R} 2$ and lemma 15. Using lemma 18 , we obtain $r k\{a, c, \beta\} \leq 2$. As $r k\{a, c\}=2$ (because $r k\{a, b, c\}=3$ ), we conclude $r k\{a, c, \beta\}=2$. Proofs for $\alpha$ and $\gamma$ are the same. 